\title{
Seletividade de Herbicidas Aplicados nas Gramas SANTo Agostinho E Esmeralda ${ }^{1}$
}

\author{
Selectivity of Herbicides Applied on ST. Augustinegrass and Emerald Turfs \\ COSTA, N.V. ${ }^{2}$, MARTINS, D. ${ }^{3}$, RODRIGUES, A.C.P. ${ }^{4}$ e CARDOSO, L.A. ${ }^{4}$
}

\begin{abstract}
RESUMO - Objetivou-se neste trabalho avaliar a seletividade de herbicidas aplicados nas gramas Santo Agostinho (Stenotaphrum secundatum) e Esmeralda (Zoysiajaponica) em condições de campo. O delineamento experimental utilizado foi o de blocos casualizados, com quatro repetições. As gramas foram cortadas a $3 \mathrm{~cm}$ de altura e, em seguida, foram feitas as aplicações dos herbicidas. Os tratamentos utilizados foram: testemunha sem aplicação, fluazifop-p-butil (125 $\left.\mathrm{g} \mathrm{ha}^{-1}\right)$, sethoxydim+óleo mineral (276 $\mathrm{g} \mathrm{ha}^{-1}+0,5 \% \mathrm{v} \mathrm{v}^{-1}$ de Assist), bispyribac-sodium (25 $\left.\mathrm{g} \mathrm{ha}^{-1}\right)$, chlorimuron-ethyl (15 $\left.\mathrm{g} \mathrm{ha}^{-1}\right)$, ethoxysulfuron $\left(150 \mathrm{~g} \mathrm{ha}^{-1}\right)$, halosulfuron $\left(112,5 \mathrm{~g} \mathrm{ha}^{-1}\right)$, iodosulfuron-methyl $\left(10 \mathrm{~g} \mathrm{ha}^{-1}\right)$, metsulfuron-methyl $\left(2,4 \mathrm{~g} \mathrm{ha}^{-1}\right)$, nicosulfuron (125 $\left.\mathrm{g} \mathrm{ha}^{-1}\right)$, pyrithiobac-sodium (140 $\left.\mathrm{g} \mathrm{ha}^{-1}\right)$, trifloxysulfuron-sodium $\left(22,5 \mathrm{~g} \mathrm{ha}^{-1}\right), 2,4-\mathrm{D}$ (720 $\left.\mathrm{g} \mathrm{ha}^{-1}\right)$, quinclorac $\left(375 \mathrm{~g} \mathrm{ha}^{-1}\right)$, atrazina $\left(1.250 \mathrm{~g} \mathrm{ha}^{-1}\right)$, bentazon $\left(600 \mathrm{~g} \mathrm{ha}^{-1}\right)$, linuron (1.350 $\left.\mathrm{g} \mathrm{ha}^{-1}\right)$, fomesafen $\left(187,5 \mathrm{~g} \mathrm{ha}^{-1}\right)$, lactofen $\left(120 \mathrm{~g} \mathrm{ha}^{-1}\right)$, oxadiazon $\left(600 \mathrm{~g} \mathrm{ha}^{-1}\right) \mathrm{e}$ oxyfluorfen $\left(720 \mathrm{~g} \mathrm{ha}^{-1}\right)$. Os herbicidas que apresentaram potencial de seletividade para o gramado de S. secundatum foram: os inibidores da ALS chlorimuron-ethyl, ethoxysulfuron, halosulfuron, iodosulfuron-methyl e metsulfuron-methyl, o mimetizador de auxina 2,4-D, os inibidores do fotossistema II atrazina e bentazon, bem como os inibidores da Protox fomesafen, lactofen e o oxadiazon. Para o gramado de $Z$. japonica, os herbicidas que apresentaram potencial de seletividade foram: os inibidores da ALS chlorimuron-ethyl, ethoxysulfuron, halosulfuron, metsulfuron-methyl e nicosulfuron, os mimetizadores de auxina 2,4-D e quinclorac, os inibidores do fotossistema II atrazina e bentazon, além dos inibidores da Protox fomesafen, lactofen e o oxadiazon.
\end{abstract}

Palavras-chave: gramado, herbicida, fitotoxicidade, Stenotaphrum secundatum, Zoysia japonica.

\begin{abstract}
The objective of this study was to evaluate the selectivity of herbicides applied on turfgrasses St. Augustinegrass (Stenotaphrum secundatum) and Emerald (Zoysia japonica) under field conditions. The experimental design was randomized blocks with four replications. The turfgrasses were cut with a motorized grass cutter to the height of $3 \mathrm{~cm}$, followed by herbicide application. The treatments were: control, fluazifop-p-butyl $(125 \mathrm{~g} \mathrm{ha}-1)$, sethoxydim+mineral oil

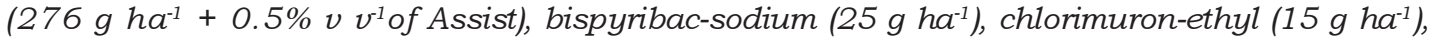
ethoxysulfuron (150 $\mathrm{g} \mathrm{ha}^{-1}$ ), halosulfuron (112.5 $\left.\mathrm{g} \mathrm{ha}^{-1}\right)$, iodosulfuron-methyl $(10 \mathrm{~g} \mathrm{ha}-1$, metsulfuron-

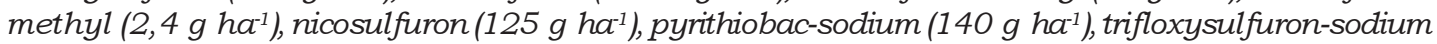

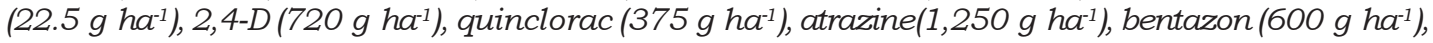
linuron (1,350 $\left.\mathrm{g} \mathrm{ha}^{-1}\right)$, fomesafen (187.5 $\mathrm{g} \mathrm{ha}-1$ ), lactofen $\left(120 \mathrm{~g} \mathrm{ha}^{-1}\right)$, oxadiazon (600 $\left.\mathrm{g} \mathrm{ha}^{-1}\right)$ and oxyfluorfen $\left(720 \mathrm{~g} \mathrm{ha}^{-1}\right)$. The following herbicides were found to have potential for selectivity of S. secundatum: ALS-inhibitors chlorimuron-ethyl, ethoxysulfuron, halosulfuron, iodosulfuron-methyl and metsulfuron-methyl, mimicking of auxin 2.4-D, the inhibitors of the atrazine and bentazon photosystem II and the inhibitors Protox fomesafem, lactofen and oxadiazon. Forturfgrass $\boldsymbol{Z}$. japonica, the following herbicides showed potential for selectivity: the ALS-inhibitors chlorimuron-ethyl, ethoxysulfuron, halosulfuron, metsulfuron-methyl and nicosulfuron, mimicking of auxin 2.4-D and quinclorac, the inhibitors of the atrazine and bentazon photosystem II and the inhibitors Protox fomesafen, lactofen and oxadiazon.
\end{abstract}

Keywords: turfgrass, herbicide, phytotoxicity, Stenotaphrum secundatum, Zoysia japonica.

Recebido para publicação em 1.3.2009 e na forma revisada em 12.3.2010.

2 Professor Adjunto da Unioeste/CCA, Marechal Cândido Rondon-PR, <neumarciovc@hotmail.com>; ${ }^{3}$ Professor Adjunto do Dep. de Produção Vegetal - FCA/UNESP, Fazenda Lageado, Caixa Postal 237, 18603-970 Botucatu-SP; ${ }^{4}$ Doutorando em Agronomia do Dep. de Produção Vegetal, FCA/UNESP, Botucatu-SP.

Planta Daninha, Viçosa-MG, v. 28, n. 1, p. 139-148, 2010 


\section{INTRODUÇÃO}

Entre as gramas recomendadas para clima quente em produção no Brasil, destacam-se as espécies Santo Agostinho (Stenotaphrum secundatum) e Esmeralda (Zoysia japonica) (Pimenta, 2003).

Essas espécies podem ser utilizadas em obras públicas (rodovias, praças, prédios públicos, etc.), parques industriais (taludes e encostas), áreas esportivas (campos de futebol, golfe, beisebol, polo, tênis, etc.), assim como em áreas residenciais (jardins). Contudo, a interferência das plantas daninhas pode prejudicar a formação, condução e estética dos gramados, além de elas competirem por água, luz, nutrientes e espaço físico, chegando em alguns casos a dizimá-los por completo (Busey, 2003; Freitas et al., 2003).

Apesar de os gramados tolerarem a competição das plantas daninhas em baixos niveis durante o seu estabelecimento, a utilização de herbicidas frequentemente torna-se necessária para a manutenção do controle das plantas daninhas (Johnson \& Carrow, 1999).

Destaca-se que o uso de roçadas no controle de plantas daninhas pode selecionar espécies com alta capacidade de rebrota e exigir maior frequência de roçadas, a exemplo das espécies Desmodium incanum e Zornia latifolia, enquanto o arranque manual em áreas extensas com alto nível de infestação torna-se inviável, por apresentar custo elevado e pela dificuldade operacional (Freitas et al., 2003).

Dessa forma, o controle químico por meio de herbicidas destaca-se como alternativa para o manejo das plantas daninhas, por contribuir para a redução dos custos de manutenção e prolongar a vida útil do gramado. É importante salientar que a utilização de herbicidas não seletivos pode provocar injúrias nos gramados e dificultar o estabelecimento na área, resultando em mais problemas com as plantas daninhas (Fagerness et al., 2002; Smith \& Callahan, 1968 citado por Busey, 2003).

No entanto, Christoffoleti \& Aranda (2001) ressaltam que não existe uma regra única de recomendação de herbicidas, sendo, portanto, necessárias recomendações diferenciadas, dependendo do nivel de toxicidade tolerado pelo gramado. Do mesmo modo, Busey (2003) e Busey \& Johnston (2006) destacam a importância do manejo integrado das plantas daninhas em gramados; para seu sucesso, devemse identificar detalhadamente os efeitos das interações dos manejos cultural e químico, uma vez que essas práticas podem ser complementares, além de proporcionarem maior flexibilidade para as tomadas de decisão.

Os herbicidas também podem ser utilizados em gramados como reguladores vegetais, com o objetivo de inibir o crescimento vegetativo das plantas, bem como inibir a emissão das inflorescências (Maciel et al., 2006; Costa et al., 2009).

No Brasil, apenas o herbicida 2,4-D é registrado para utilização em gramados (Rodrigues \& Almeida, 2005). Dessa forma, a identificação de herbicidas seletivos e que apresentam mecanismos de ação distintos torna-se fundamental para o desenvolvimento de programas de manejo das plantas daninhas em gramados para as condições brasileiras, além de evitar a seleção de espécies resistentes a herbicidas.

Em outros países, vários pesquisadores destacaram a viabilidade do uso de herbicidas pré e pós-emergentes em vários tipos de gramados, a exemplo de atrazina, oxadiazon, quinclorac, sethoxydim, entre outros, bem como a possibilidade do emprego desses produtos em mistura em tanque, de forma a maximizar os efeitos dos diferentes mecanismos de ação no controle das plantas daninhas (Turner et al., 1990; McCarty et al., 1995; Johnson \& Carrow, 1999; Brecke et al., 2001; Fagerness et al., 2002).

Assim, o objetivo do presente trabalho foi o de avaliar a seletividade de diferentes herbicidas aplicados na gramas Santo Agostinho ( $S$. secundatum) e Esmeralda ( $Z$. japonica) em condições de campo.

\section{MATERIAL E MÉTODOS}

O estudo foi conduzido no Núcleo de Pesquisas Avançadas em Matologia - NUPAM, do Departamento de Produção Vegetal, pertencente à Faculdade de Ciências Agronômicas de Botucatu/UNESP. O local do experimento 
apresenta as seguintes coordenadas geográficas: latitude de $22^{\circ} 07^{\prime} 56^{\prime \prime} \mathrm{S}$, longitude de $74^{\circ} 66^{\prime} 84^{\prime}$ W Gr. e altitude de $762 \mathrm{~m}$. Foram avaliadas as espécies de grama Santo Agostinho ( $S$. secundatum) e Esmeralda ( $Z$. japonica). Essas espécies foram plantadas em tabuleiros individuais e estavam com 26 meses de idade no momento da aplicação dos tratamentos. Em cada espécie de grama foi utilizado o delineamento experimental em blocos ao acaso, com quatro repetições. As parcelas foram constituídas de $2 \mathrm{~m}$ de largura por $2 \mathrm{~m}$ de comprimento. Na Tabela 1 são mostrados os herbicidas, avaliados por nome comum, nome comercial, mecanismo de ação e dose utilizada.

Em 12/1/2006, os gramados foram cortados à altura de $3 \mathrm{~cm}$ com auxílio de um aparador de grama motorizado e, após dois dias, foram realizadas as aplicações dos tratamentos. As aplicações foram realizadas no período da manhã, em condições de temperatura de $27^{\circ} \mathrm{C}$, solo úmido e umidade relativa do ar em torno de $64 \%$. Foi utilizado um pulverizador costal, pressurizado a $\mathrm{CO}_{2}$ e munido de barra com quatro pontas de jato plano DG 11002 VS, espaçados entre si de $50 \mathrm{~cm}$, sendo o consumo de calda equivalente a $200 \mathrm{~L} \mathrm{ha}^{-1}$. Durante a aplicação, as parcelas foram protegidas lateralmente com biombos de lona plástica, com o objetivo de evitar deriva de calda pulverizada para as parcelas adjacentes.

As avaliações de fitointoxicação nos gramados foram feitas de forma visual, utilizando-se uma escala de percentual de notas, em que 0 (zero) corresponde a nenhuma injúria demonstrada pela planta e 100 (cem) à morte das plantas (SBCPD, 1995). Dessa forma, para evitar grandes prejuízos ao aspecto visual do gramado e facilitar a interpretação dos resultados de fitointoxicação, foram consideradas como sintomas leves as notas inferiores a $10,0 \%$; como sintomas moderados, as notas entre 10,1 e $20,0 \%$; e como sintomas severos e não aceitáveis esteticamente, as notas superiores a $20,0 \%$. As avaliações foram realizadas aos 3, 7, 14, 26 e 49 dias após aplicação dos herbicidas. Os critérios usados para o estabelecimento das notas foram: inibição do crescimento, quantidade e uniformidade das injúrias, capacidade de rebrota das plantas e quantidade de plantas mortas.
Os caracteres morfológicos avaliados foram: número e comprimento de inflorescência, altura da grama e massa seca da parte aérea. As avaliações foram realizadas no final do experimento, aos 49 dias após a aplicação dos herbicidas.

O número de inflorescências foi avaliado por amostragem, com auxílio de um quadro de $0,25 \mathrm{~m}^{2}$ colocado no centro das parcelas, e o comprimento das inflorescências foi determinado com auxílio de uma régua graduada, medindo-se a distância entre o solo e as extremidades, amostrando-se apenas a inflorescência mais alta do centro de cada parcela. As avaliações da altura da grama foram feitas com auxílio de duas réguas graduadas, sendo posicionadas, no momento da avaliação, perpendicular e horizontalmente ao solo (em forma de cruz) em apenas um ponto de cada parcela, medindo-se a distância entre o solo e a extremidade da folha mais alta. A régua na horizontal tinha como função facilitar a determinação da folha mais alta do centro da parcela.

Tabela 1 - Herbicidas avaliados, indicados por nome comum, nome comercial, mecanismo de ação e dose utilizada. Botucatu-SP, 2006

\begin{tabular}{|c|c|c|c|}
\hline Nome comum & Nome comercial & $\begin{array}{l}\text { Mecanismo de } \\
\text { ação }\end{array}$ & $\frac{\text { Dose }}{\left(\text { g i.a. ha }^{-1}\right)}$ \\
\hline Testemunha & -- & -- & --- \\
\hline fluazifop-p-butil & Fusilade 125 & Accase & 125,0 \\
\hline sethoxydim $^{1 /}$ & Poast & Accase & 276,0 \\
\hline bispyribac-sodium & Nominee $400 \mathrm{SC}$ & ALS & 25,0 \\
\hline chlorimuron-ethyl & Smart & ALS & 15,0 \\
\hline ethoxysulfuron & Gladium & ALS & 150,0 \\
\hline halosulfuron & Sempra & ALS & 112,5 \\
\hline iodosulfuron-methyl & Hussar & ALS & 10,0 \\
\hline metsulfuron-methyl & Ally & ALS & 2,4 \\
\hline nicosulfuron & Sanson $40 \mathrm{SC}$ & ALS & 40,0 \\
\hline pyrithiobac-sodium & Staple 280 CS & ALS & 140,0 \\
\hline trifloxysulfuron-sodium & Envoke & ALS & 22,5 \\
\hline $2,4-\mathrm{D}$ & U46 D-Fluid & Auxina & 720,0 \\
\hline quinclorac & Facet PM & Auxina & 375,0 \\
\hline atrazina & Gesaprim 500 & Fotossistema II & 1250,0 \\
\hline bentazon & Basagran 600 & Fotossistema II & 600,0 \\
\hline linuron & Afalon SC & Fotossistema II & 1350,0 \\
\hline fomesafen & Flex & Protox & 187,5 \\
\hline lactofen & Cobra & Protox & 120,0 \\
\hline oxadiazon & Ronstar SC & Protox & 600,0 \\
\hline oxyfluorfen & Goal BR & Protox & 720,0 \\
\hline
\end{tabular}

${ }^{1 /}$ Adicionou-se o adjuvante Assist $\left(0,5 \% \mathrm{v} \mathrm{v}^{-1}\right)$ à calda de aplicação. 
A massa seca da parte aérea produzida pelas gramas foi determinada, coletando-se a massa vegetal produzida na parcela após o corte da grama a $3 \mathrm{~cm}$ de altura, com auxílio de um aparador de grama motorizado, para posterior secagem por 96 horas em estufa de circulação forçada de ar a $65^{\circ} \mathrm{C}$.

Os resultados foram submetidos à análise de variância pelo teste $\mathrm{F}$, sendo analisados estatisticamente pelo teste de média LSD a $5 \%$ de probabilidade. Os dados originais, em número de inflorescências e porcentagem de fitotoxicidade, foram transformados utilizando-se $\sqrt{x}+0,5$ e arco seno $\sqrt{x} / 100$, respectivamente.

\section{RESULTADOS E DISCUSSÃO}

\section{S. secundatum}

Verificou-se que todos os herbicidas promoveram intoxicação nas plantas a partir do 3 DAA, sendo os maiores valores encontrados nas parcelas tratadas com trifloxysulfuronsodium e oxyfluorfen, com médias na ordem de 13,8 e 20,0\%, respectivamente (Tabela 2).

Analisando os herbicidas inibidores da Accase (acetil-coenzima-A carboxilase), observou-se que os sintomas de intoxicação foram considerados severos, com valores de $31,3 \%$ para o herbicida sethoxydim aos 14 DAA e de $80,0 \%$ para o fluazifop-p-butil aos 26 DAA, sendo que este proporcionou sintomas no gramado por maior período de tempo, em relação aos demais herbicidas avaliados. Willard et al. (1990) verificaram também em condições de campo que a grama $S$. secundatum foi suscetível à aplicação do sethoxydim $\left(280 \mathrm{~g} \mathrm{ha}^{-1}\right) \mathrm{e}$ do fluazifop-p-butil (280 $\mathrm{g}$ ha ${ }^{1}$ ), uma vez que esses herbicidas reduziram o processo de fotossintese da grama em cerca de 40,0 e 38,0\%, respectivamente, aos 12 DAA.

O sethoxydim e o fluazifop-p-butil são herbicidas gramicidas utilizados nas culturas de algodão, batata, feijão, soja, tabaco, milho, entre outras (Gazziero et al., 2004; Rodrigues \& Almeida, 2005; Santos et al., 2006; Dvoranen et al., 2008). Esse aspecto pode explicar os sintomas severos de intoxicação no gramado, porém doses menores ou a mistura com outros produtos químicos (protetores) podem tornar esses herbicidas mais seletivos.
Entretanto, para os inibidores da ALS (acetolactato sintase), o metsulfuron-methyl, o halosulfuron, o ethoxysulfuron e o chlorimuron-ethyl, os sintomas foram considerados leves, persistindo por no máximo 7 DAA; contudo, para o iodosulfuron-methyl, os sintomas mais elevados não ultrapassaram 13,8\% por um período de até 14 DAA. Dessa maneira, esses niveis de intoxicação podem ser tolerados em gramados, uma vez que ocorreram por um periodo curto de tempo, além do fato de a utilização de irrigação, fertilizantes ou de corantes artificiais pode contribuir para reduzir o período do desaparecimento dos sintomas de fitointoxicação. Para o bispyribac-sodium, os sintomas foram considerados moderados, porém persistindo por um periodo de até 26 DAA. Os demais herbicidas pertencentes a esse mecanismo de ação promoveram fitointoxicação severa (> 20,0\%) em comparação aos herbicidas citados anteriormente, podendo prejudicar o aspecto visual do gramado por um período de até 26 DAA (Tabela 2).

Dos mimetizadores de auxina, o 2,4-D destacou-se entre os herbicidas que apresentaram as menores médias de fitotoxicidade $(\leq 2,0 \%)$, enquanto o quinclorac proporcionou sintomas de até $37,5 \%$ aos 14 DAA. Para os inibidores do fotossistema II, apenas o linuron mostrou sintomas de fitointoxicação em torno de $55,0 \%$; entretanto, o bentazon e a atrazina proporcionaram sintomas leves e inferiores a 1,5 e $2,0 \%$, respectivamente, por um período de até 7 DAA.

Da mesma forma, para os inibidores da Protox (protoporfirinogênio oxidase), oxadiazon, lactofen e fomesafen, os valores mais elevados de fitointoxicação foram observados aos 14 DAA, sendo considerados moderados e inferiores a $10,5 \%$, exceto para o oxyflourfen, que apresentou $47,5 \%$ de fitointoxicação no mesmo periodo.

No geral, os herbicidas chlorimuron-ethyl, ethoxysulfuron, halosulfuron, iodosulturonmethyl, metsulfuron-methyl, 2,4-D, atrazina e bentazon foram os que apresentaram sintomas de fitointoxicação leves a moderados, por um período máximo de 14 DAA; a partir dessa data, os sintomas desapareceram completamente. No entanto, os herbicidas fluazifop-pbutil, sethoxydim, nicosulfuron, pyrithiobacsodium, trifloxysulfuron-sodium, quinclorac, 
Tabela 2 - Porcentagem de fitointoxicação em plantas de S. secundatum, após a aplicação de diferentes herbicidas. Botucatu-SP, 2006

\begin{tabular}{|c|c|c|c|c|c|c|c|}
\hline \multirow{2}{*}{ Tratamento } & \multirow{2}{*}{$\frac{\text { Dose }}{\left(\text { g i.a. } \mathrm{ha}^{-1}\right)}$} & \multirow{2}{*}{$\begin{array}{l}\text { Mecanismo } \\
\text { de ação }\end{array}$} & \multicolumn{5}{|c|}{ Dias após a aplicação ${ }^{\underline{2}}$} \\
\hline & & & 3 & 7 & 14 & 26 & 49 \\
\hline Testemunha & --- & --- & $0,0(0,0) \mathrm{h}$ & $0,0(0,0) \mathrm{j}$ & $0,0(0,0) \mathrm{i}$ & $0,0(0,0) \mathrm{f}$ & $0,0(0,0) \mathrm{b}$ \\
\hline fluazifop-p-butil & 125,0 & Accase & $2,8(9,3)$ cdef & $46,3(42,8)$ a & $61,3(51,7) a$ & $80,0(63,5)$ a & $30,0(33,1) \mathrm{a}$ \\
\hline sethoxydim $^{1 /}$ & 276,0 & Accase & $3,0(9,3)$ cdef & $13,8(21,0)$ def & $31,3(33,6) \mathrm{cd}$ & $18,8(24,4)$ bc & $0,0(0,0) \mathrm{b}$ \\
\hline bispyribac-sodium & 25,0 & ALS & $2,0(7,8)$ efg & 9,3 $(14,4)$ efgh & $18,8(24,1) \mathrm{fg}$ & $4,6(12,4) \mathrm{e}$ & $0,0(0,0) \mathrm{b}$ \\
\hline chlorimuron-ethyl & 15,0 & ALS & $2,0(8,1) \mathrm{def}$ & $4,8(11,9)$ ghi & $0,0(0,0) \mathrm{i}$ & $0,0(0,0) \mathrm{f}$ & $0,0(0,0) \mathrm{b}$ \\
\hline ethoxysulfuron & 150,0 & ALS & $2,0(8,1) \mathrm{def}$ & $6,0(13,0)$ fghi & $0,0(0,0) \mathrm{i}$ & $0,0(0,0) \mathrm{f}$ & $0,0(0,0) \mathrm{b}$ \\
\hline halosulfuron & 112,5 & ALS & $1,5(9,6) \mathrm{fg}$ & $1,0(5,7) \mathrm{ij}$ & $0,0(0,0) \mathrm{i}$ & $0,0(0,0) \mathrm{f}$ & $0,0(0,0) \mathrm{b}$ \\
\hline iodosulfuron-methyl & 10,0 & ALS & $2,3(8,6)$ cdef & $13,8(21,6) \mathrm{de}$ & $7,5(15,4) \mathrm{gh}$ & $0,0(0,0) \mathrm{f}$ & $0,0(0,0) \mathrm{b}$ \\
\hline metsulfuron-methyl & 2,4 & ALS & $1,3(5,5) \mathrm{fg}$ & $1,0(5,7) \mathrm{ij}$ & $0,0(0,0) \mathrm{i}$ & $0,0(0,0) \mathrm{f}$ & $0,0(0,0) \mathrm{b}$ \\
\hline nicosulfuron & 40,0 & ALS & $1,8(6,3) \mathrm{fg}$ & $8,0(14,3)$ efgh & $21,8(27,6)$ ef & $19,0(25,8) \mathrm{b}$ & $0,0(0,0) \mathrm{b}$ \\
\hline pyrithiobac-sodium & 140,0 & ALS & $2,0(8,1)$ def & $30,0(32,8)$ bc & $23,8(28,1)$ ef & $12,5(17,8)$ cde & $0,0(0,0) \mathrm{b}$ \\
\hline trifloxysulfuron-sodium & 22,5 & ALS & $13,8(21,6) b$ & $41,3(39,9) a b$ & $26,3(30,5)$ cde & $13,8(20,6)$ bcd & $0,0(0,0) \mathrm{b}$ \\
\hline $2,4-\mathrm{D}$ & 720,0 & Auxina & $2,0(8,0)$ ef & $1,3(6,3)$ hij & $0,0(0,0) \mathrm{i}$ & $0,0(0,0) \mathrm{f}$ & $0,0(0,0) \mathrm{b}$ \\
\hline quinclorac & 375,0 & Auxina & $5,3(12,5) \mathrm{cd}$ & $23,8(29,1) \mathrm{cd}$ & $37,5(37,6) b c$ & $6,3(12,4) \mathrm{e}$ & $0,0(0,0) \mathrm{b}$ \\
\hline atrazina & $1.250,0$ & Fotossistema II & $1,3(5,5) \mathrm{fg}$ & 2,0 $(7,5)$ ghij & $0,0(0,0) \mathrm{i}$ & $0,0(0,0) \mathrm{f}$ & $0,0(0,0) \mathrm{b}$ \\
\hline bentazon & 600,0 & Fotossistema II & $0,8(3,5) \mathrm{gh}$ & 1,5 $(6,8)$ ghij & $0,0(0,0) \mathrm{i}$ & $0,0(0,0) \mathrm{f}$ & $0,0(0,0) \mathrm{b}$ \\
\hline linuron & $1.350,0$ & Fotossistema II & $5,3(12,9) \mathrm{c}$ & $55,0(47,9) \mathrm{a}$ & $31,3(33,8) \mathrm{cd}$ & $0,0(0,0) \mathrm{f}$ & $0,0(0,0) \mathrm{b}$ \\
\hline fomesafen & 187,5 & \begin{tabular}{|l} 
Protox \\
\end{tabular} & $2,5(9,0)$ cdef & \begin{tabular}{|l}
$3,5(9,5)$ ghi \\
\end{tabular} & $10,5(18,7) \mathrm{gh}$ & $0,0(0,0) \mathrm{f}$ & $0,0(0,0) \mathrm{b}$ \\
\hline lactofen & 120,0 & Protox & $1,8(7,4)$ efg & $1,0(5,7) \mathrm{ij}$ & $6,3(12,4) \mathrm{h}$ & $0,0(0,0) \mathrm{f}$ & $0,0(0,0) \mathrm{b}$ \\
\hline oxadiazon & 600,0 & Protox & $4,5(11,6)$ cde & $9,3(15,2)$ efg & $10,0(15,6) \mathrm{gh}$ & $0,0(0,0) \mathrm{f}$ & $0,0(0,0) \mathrm{b}$ \\
\hline oxyfluorfen & 720,0 & Protox & $20,0(26,5) \mathrm{a}$ & $45,0(42,1) \mathrm{a}$ & $47,5(43,6) \mathrm{ab}$ & $7,8(16,1) \mathrm{de}$ & $0,0(0,0) \mathrm{b}$ \\
\hline $\mathrm{F}_{\text {Tratamento }}$ & & & $1,925^{*}$ & $4,263^{* *}$ & $30,154 * *$ & $43,613 * *$ & $209,552 * *$ \\
\hline $\mathrm{F}_{\text {Bloco }}$ & & & $1,064^{\mathrm{ns}}$ & $4,295^{* *}$ & $1,705^{\mathrm{ns}}$ & $0,946^{\mathrm{ns}}$ & $1,000^{\mathrm{ns}}$ \\
\hline $\mathrm{CV}(\%)$ & & & 20,20 & 21,27 & 34,61 & 51,21 & 63,31 \\
\hline d.m.s. & & & 1,609 & 1,808 & 8,682 & 6,653 & 1,411 \\
\hline
\end{tabular}

${ }^{1 /}$ Adicionou-se o adjuvante Assist $\left(0,5 \% \mathrm{v} \mathrm{v}^{-1}\right)$ à calda de aplicação. ${ }^{2 /}$ dados originais foram transformados em arco seno $\sqrt{\mathrm{x}} / 100$ (entre parênteses). Médias seguidas de mesma letra, nas linhas, não diferem estatisticamente entre si a $5 \%$ de probabilidade pelo teste LSD. ** significativo a $1 \%$ de probabilidade; $*$ significativo a $5 \%$ de probabilidade; ${ }^{\text {ns }}$ não significativo.

linuron e oxyfluorfen promoveram fitointoxicações consideradas severas, por um periodo de 26 a 49 DAA. O oxadiazon e o lactofen apresentaram sintomas de fitointoxicação considerados leves e inferiores a 10,0 e 6,3\%, respectivamente, por somente 14 DAA. Ressalta-se que aos 49 DAA o gramado não mostrou sintomas de fitointoxicação promovidos pelos herbicidas avaliados, com exceção do fluazifopp-butil.

Pode-se verificar que, para a densidade de inflorescências, apenas o fluazifop-p-butil promoveu redução $(91,5 \%)$ em relação à testemunha. Entretanto, o 2,4-D (Auxina), a atrazina e o bentazon (fotossistema II), além do fomesafen, oxadiazon e oxyfluorfen (Protox), estimularam a emissão de inflorescências em torno de 160,$7 ; 358,4 ; 122,5 ; 158,4 ; 202$,2; e $264,0 \%$, respectivamente, quando se compara com a testemunha (Tabela 3). Verificou-se ainda que todos os inibidores da ALS não influenciaram a densidade de inflorescências, juntamente com o sethoxydim (Accase), o quinclorac (auxina), o linuron (fotossistema II) e o lactofen (Protox).

Quanto à altura das inflorescências, apenas o nicosulfuron, o 2,4-D e o bispyribacsodium promoveram reduções na ordem de $40,0,28,6$ e $24,8 \%$, respectivamente, em relação à testemunha.

Com relação à altura da grama, notou-se que os herbicidas sethoxydim, nicosulfuron, halosulfuron, quinclorac, bentazon e lactofen retardaram o crescimento do gramado em torno de 26,$9 ; 26,9 ; 32,7 ; 26,9 ; 25,0$; e 30,8\%, respectivamente, no período de 49 DAA. Esse valores representam em média cerca de $2 \mathrm{~cm}$ a menos em relação à altura das plantas da testemunha. Assim, pode-se inferir que os herbicidas halosulfuron, bentazon e lactofen podem auxiliar no manejo da altura do gramado de $S$. secundatum, sem afetar severamente o aspecto visual, uma vez que apresentaram sintomas de fitotoxicidade inferiores a $2,0 \%$ por até 7 DAA, bem como o lactofen, que proporcionou sintomas leves $(\leq 6,3 \%)$ por até 14 DAA (Tabela 2$)$. 
Apenas os inibidores da ALS chlorimuronethyl, ethoxysulfuron e iodosulfuron-methyl não reduziram a massa seca das plantas aos 49 DAA; contudo, o chlorimuron-ethyl e o ethoxysulfuron apresentaram sintomas leves a moderados de fitotoxicidade no gramado por apenas 7 DAA, enquanto os sintomas do iodosulfuron-methyl $(\leq 13,8 \%)$ persistiram por apenas 14 DAA (Tabelas 2 e 3).

Considerando os herbicidas que proporcionaram fitointoxicação leve $(\leq 10,0 \%)$ por no máximo uma semana e que reduziram a massa seca das plantas entre 50,0 e $70,0 \%$ aos 49 DAA, destacaram-se o halosulfuron, o metsulfuron-methyl, o 2,4-D, o atrazina e o bentazon. Esses resultados corroboram os obtidos por Christoffoleti \& Aranda (2001), que avaliaram a seletividade dos herbicidas halosulfuron (112,5 $\left.\mathrm{g} \mathrm{ha}^{-1}\right)$ e 2,4-D (2.010 $\left.\mathrm{g} \mathrm{ha}^{-1}\right)$ na grama $S$. secundatum e observaram sintomas de fitotoxicidade considerados entre leve e moderado aos 50 DAA. Entretanto, esses pesquisadores verificaram que o oxadiazon (1.000 $\mathrm{g} \mathrm{ha}^{-1}$ ) promoveu sintomas considerados severos para o mesmo período de avaliação.

Da mesma forma, McCarty et al. (1995) observaram sintomas leves promovidos pelos herbicidas atrazina (2.200 $\left.\mathrm{g} \mathrm{ha}^{-1}\right)$ e oxadiazon (3.400 $\mathrm{g} \mathrm{ha}^{-1}$ ) quando aplicados em pré-emergência na grama $S$. secundatum por um periodo de até 14 DAA. Entretanto, Shim \& Johnson (1992) verificaram que o oxadiazon na dose de $3.400 \mathrm{~g} \mathrm{ha}^{-1}$ promoveu fitointoxicação de moderada a severa na grama Agrostis palustris no período de até 21 DAA. Destaca-se que esses autores consideraram as notas de fitointoxicação entre 1 e $20 \%$ como sintomas leves; entre 21 e $30 \%$, como sintomas moderados; e superiores a $30 \%$, como sintomas severos e não aceitáveis comercialmente.

Tabela 3 - Valores médios de número de inflorescência, altura da planta, altura de inflorescência e massa seca do gramado de S. secundatum, após a aplicação de diferentes herbicidas, aos 49 DAA. Botucatu-SP, 2006

\begin{tabular}{|c|c|c|c|c|c|c|}
\hline \multirow[t]{2}{*}{ Tratamento } & Dose & \multirow[t]{2}{*}{$\begin{array}{l}\text { Mecanismo } \\
\text { de ação }\end{array}$} & Inflorescência & $\begin{array}{c}\text { Altura da } \\
\text { Inflorescência }\end{array}$ & $\begin{array}{c}\text { Altura da } \\
\text { grama }\end{array}$ & \multirow{2}{*}{$\begin{array}{c}\text { Massa seca } \\
\left(\mathrm{kg} \mathrm{ha}^{-1}\right)\end{array}$} \\
\hline & $\left(\right.$ g i.a. ha $\left.{ }^{-1}\right)$ & & $\left(\text { número } \mathrm{m}^{-2}\right)^{2 /}$ & \multicolumn{2}{|c|}{$(\mathrm{cm})$} & \\
\hline Testemunha & --- & --- & $44,5(6,5) \mathrm{fg}$ & $10,5 \mathrm{cde}$ & $6,5 \mathrm{ab}$ & $1322,3 \mathrm{a}$ \\
\hline fluazifop-p-butil & 125,0 & Accase & $3,8(2,1) \mathrm{h}$ & 8,6 efgh & 5,8 abcde & 515,5 efgh \\
\hline sethoxydim $^{-1}$ & 276,0 & Accase & $20,3(4,5) \mathrm{gh}$ & 8,0 efgh & 4,8 cde & $419,9 \mathrm{~h}$ \\
\hline bispyribac-sodium & 25,0 & ALS & $52,8(7,1)$ efg & $7,9 \mathrm{fgh}$ & 5,6 abcde & $375,1 \mathrm{~h}$ \\
\hline chlorimuron-ethyl & 15,0 & ALS & $37,0(6,1) \mathrm{fg}$ & $14,1 \mathrm{a}$ & 7,0 a & $1144,3 \mathrm{ab}$ \\
\hline ethoxysulfuron & 150,0 & ALS & $44,0(6,5) \mathrm{fg}$ & 11,8 abcd & 6,0 abcd & 1016,6 abcd \\
\hline halosulfuron & 112,5 & ALS & $78,0(8,7)$ cdef & 8,6 efgh & $4,4 \mathrm{e}$ & $431,0 \mathrm{~h}$ \\
\hline iodosulfuron-methyl & 10,0 & ALS & $44,0(6,6) \mathrm{fg}$ & 11,8 abcd & 6,0 abcd & $1045,5 \mathrm{abc}$ \\
\hline metsulfuron-methyl & 2,4 & ALS & $43,0(6,4) \mathrm{fg}$ & 10,0 cdefg & 5,0 bcde & $414,9 \mathrm{~h}$ \\
\hline nicosulfuron & 40,0 & ALS & $44,0(6,6) \mathrm{fg}$ & $6,3 \mathrm{~h}$ & 4,8 cde & $442,9 \mathrm{gh}$ \\
\hline pyrithiobac-sodium & 140,0 & ALS & $44,3(6,4) \mathrm{fg}$ & 9,9 cdefg & 5,9 abcde & 835,3 bcdef \\
\hline trifloxysulfuron-sodium & 22,5 & ALS & $42,3(6,3) \mathrm{fg}$ & $13,8 \mathrm{ab}$ & $6,8 \mathrm{a}$ & 886,2 bcde \\
\hline $2,4-\mathrm{D}$ & 720,0 & Auxina & $116,0(10,5)$ bed & $7,5 \mathrm{gh}$ & 5,5 abcde & $451,2 \mathrm{gh}$ \\
\hline quinclorac & 375,0 & Auxina & $83,0(9,0)$ cdef & $11,3 \mathrm{bcd}$ & 4,8 cde & 510,6 efgh \\
\hline atrazina & $1.250,0$ & Fotossistema II & $99,0(9,8)$ bcde & $11,9 \mathrm{abc}$ & $6,1 \mathrm{abc}$ & 609,3 efgh \\
\hline bentazon & 600,0 & Fotossistema II & $204,0(13,8)$ a & 10,3 cdef & 4,9 cde & $499,5 \mathrm{fgh}$ \\
\hline linuron & $1.350,0$ & Fotossistema II & $61,0(7,7)$ def & 11,8 abcd & $6,1 \mathrm{abc}$ & 663,9 defgh \\
\hline fomesafen & 187,5 & Protox & $162,0(12,3) \mathrm{ab}$ & 8,6 efgh & 5,6 abcde & 709,6 cdefgh \\
\hline lactofen & 120,0 & Protox & $68,0(8,2)$ def & 9,3 defg & $4,5 \mathrm{de}$ & $419,6 \mathrm{~h}$ \\
\hline oxadiazon & 600,0 & Protox & $115,0(10,5)$ bcd & 9,5 cdefg & 5,5 abcde & $455,1 \mathrm{gh}$ \\
\hline oxyfluorfen & 720,0 & Protox & $134,5(11,4)$ abc & $11,5 \mathrm{bcd}$ & 6,9 a & 816,0 bcdefg \\
\hline $\mathrm{F}_{\text {Tratamento }}$ & & & $6,958^{* *}$ & $5,216^{* *}$ & $1,925^{*}$ & $4,404^{* *}$ \\
\hline$F_{\text {Bloco }}$ & & & $1,930^{\mathrm{ns}}$ & $2,766^{*}$ & $1,064^{\mathrm{ns}}$ & $0,310^{\mathrm{ns}}$ \\
\hline $\mathrm{CV}(\%)$ & & & 26,03 & 17,62 & 20,20 & 40,10 \\
\hline d.m.s. & & & 2,931 & 2,523 & 1,609 & 377,713 \\
\hline
\end{tabular}

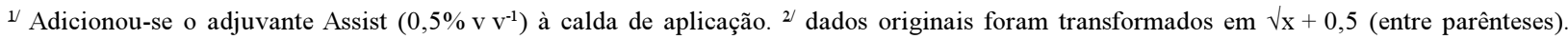
Médias seguidas de mesma letra, nas linhas, não diferem estatisticamente entre si a $5 \%$ de probabilidade pelo teste LSD.

${ }^{* *}$ significativo a $1 \%$ de probabilidade; ${ }^{*}$ significativo a $5 \%$ de probabilidade; ${ }^{\text {ns }}$ não significativo. 
Segundo Turner et al. (1990), os herbicidas atrazina $\left(2.200 \mathrm{e} 3.400 \mathrm{~g} \mathrm{ha}^{-1}\right)$, oxadiazon $\left(2.200 \mathrm{e}^{2} .500 \mathrm{~g} \mathrm{ha}^{-1}\right)$ e sethoxydim $(280 \mathrm{e}$ $560 \mathrm{~g} \mathrm{ha}^{-1}$ ) não promoveram sintomas de fitotoxicidade na grama Eremochloa ophiuroides no período de 42 DAA. Entretanto, o oxadiazon aplicado no estádio inicial de desenvolvimento da grama nas doses de 2.200 e $4.500 \mathrm{~g} \mathrm{ha}^{-1}$ apresentou 21,0 e $71,0 \%$ de fitointoxicação, respectivamente, aos 21 DAA. Esses autores afirmam ainda que esses herbicidas não reduziram a densidade e o comprimento do sistema radicular em comparação com a testemunha, independentemente do estádio de desenvolvimento da grama $E$. ophiuroides.

Observou-se que os herbicidas fomesafen, lactofen e oxadiazon proporcionaram fitointoxicação moderada $(\leq 10,5 \%)$ e reduções na massa seca da parte aérea das plantas de S. secundatum em torno de 46,3, 65,6 e 68,3\% aos 49 DAA, respectivamente. Da mesma maneira, os herbicidas fluazifop-p-butil, sethoxydim, bispyribac-sodium, nicosulfuron, pyrithiobac-sodium, trifloxysulfuron-sodium, quinclorac, linuron e oxyfluorfen também reduziram a massa seca, porém prejudicaram o aspecto visual do gramado, por um período de até 26 DAA (Tabelas 2 e 3). De acordo com Willard et al. (1990), a aplicação do sethoxydim (280 $\left.\mathrm{g} \mathrm{ha}^{-1}\right)$ e do fluazifop-p-butil (280 $\left.\mathrm{g} \mathrm{ha}^{-1}\right)$ reduziu a massa seca da grama $S$. secundatum em torno de 68,0 e $70,0 \%$, respectivamente, aos 12 DAA.

\section{Z. japonica}

Pode-se observar que, a partir dos 3 DAA, todos os herbicidas promoveram fitointoxicação no gramado, destacando-se o oxyfluorfen, que apresentou sintomas severos, na ordem de $22,5 \%$ (Tabela 4 ).

Verificou-se, para os inibidores da Accase, que os resultados foram semelhantes aos observados para o gramado de $S$. secundatum, com sintomas considerados de moderados a severos, com valores superiores a 16,3 e 50,0\% para os herbicidas sethoxydim e fluazifop-pbutil, respectivamente, a partir dos 7 DAA. Contudo, os sintomas proporcionados pelo fluazifop-p-butil prolongaram-se até os 26 DAA.

Quanto aos inibidores da ALS, somente o halosulfuron mostrou média de fitointoxicação inferior a 5,0\% e por até 7 DAA. Entretanto, o chlorimuron-ethyl, o ethoxysulfuron, o metsulfuron-methyl e o nicosulfuron apresentaram sintomas moderados de fitointoxicação, os quais desapareceram completamente aos 26 DAA. Os demais herbicidas pertencentes aos inibidores da ALS apresentaram fitointoxicação severa (> 20,0\%), persistindo os sintomas por até 14 DAA, com exceção do pyrithiobacsodium.

Os mimetizadores de auxina, 2,4-D e quinclorac, destacaram-se entre os herbicidas que apresentaram médias leves de fitointoxicação $(\leq 4,0 \%)$, com o desaparecimento dos sintomas por completo a partir dos 14 DAA. É importante ressaltar que o quinclorac prejudicou severamente o aspecto visual da grama $S$. secundatum por um período de até 26 DAA (Tabela 2).

Com relação aos inibidores do fotossistema II, apenas o bentazon apresentou sintomas de fitointoxicação leves por até 7 DAA. A atrazina proporcionou sintomas com valor máximo de $15,0 \%$ de fitointoxicação por um período de até 14 DAA. Contudo, o linuron mostrou médias severas $(\geq 60,0 \%)$ por um período de 14 DAA.

Entre os inibidores da Protox, o fomesafen foi o herbicida que mais se destacou, visto que proporcionou fitointoxicação inferior a 3,0\% por um período máximo de 7 DAA. O oxadiazon e o lactofen apresentaram sintomas moderados, em torno de 11,8 e $16,8 \%$, respectivamente, aos 7 DAA; os sintomas desapareceram completamente a partir dos 26 DAA. Os sintomas proporcionados pelo oxyflourfen em $Z$. japonica foram considerados severos, resultado semelhante ao verificado na grama S. secundatum. Já Johnson \& Carrow (1999) relataram que a aplicação em pré-emergência do oxadiazon ( $3.400 \mathrm{~g} \mathrm{ha}^{-1}$ ) não prejudicou a qualidade estética de oito cultivares de grama Zoysia por um período de até 77 DAA.

Assim, entre os herbicidas avaliados, os que proporcionaram sintomas de fitointoxicação leves $(\leq 10,0 \%)$ por um período máximo de 7 DAA foram: halosulfuron, 2,4-D, quinclorac, bentazon e fomesafen. Contudo, os herbicidas fluazifop-p-butil, bispyribac-sodium, pyrithiobac-sodium, iodosulfuron-methyl, trifloxysulfuron-sodium, linuron e oxyfluorfen foram os que promoveram fitointoxicação severa ( $\geq 20,0 \%$ ) por um período de até 26 DAA. Ressalta-se que, aos 49 DAA, o gramado não 
Tabela 4 - Porcentagem de fitointoxicação de plantas de Z. japonica, após a aplicação de diferentes herbicidas. Botucatu-SP, 2006

\begin{tabular}{|c|c|c|c|c|c|c|c|}
\hline \multirow{2}{*}{ Tratamento } & \multirow{2}{*}{$\frac{\text { Dose }}{\left(\mathrm{g} \text { i.a. } \mathrm{ha}^{-1}\right)}$} & \multirow{2}{*}{$\begin{array}{l}\text { Mecanismo } \\
\text { de ação }\end{array}$} & \multicolumn{5}{|c|}{ Dias após a aplicação $0^{\underline{2}}$} \\
\hline & & & 3 & 7 & 14 & 26 & 49 \\
\hline Testemunha & --- & --- & $0,0(0,0) \mathrm{e}$ & $0,0(0,0) 1$ & $0,0(0,0) \mathrm{g}$ & $0,0(0,0) \mathrm{c}$ & 0,0 \\
\hline fluazifop-p-butil & 125,0 & Accase & $5,3(11,3)$ bcd & $50,0(45,0)$ ab & $60,0(50,9)$ a & $40,0(31,7) \mathrm{a}$ & 0,0 \\
\hline sethoxydim ${ }^{1 /}$ & 276,0 & Accase & $2,8(9,4)$ bcd & $16,3(23,6)$ efg & $18,8(24,1) \mathrm{cd}$ & $0,0(0,0) \mathrm{c}$ & 0,0 \\
\hline bispyribac-sodium & 25,0 & ALS & $6,8(14,1) \mathrm{b}$ & $30,0(33,1) \mathrm{cd}$ & $23,8(28,4) \mathrm{c}$ & $0,0(0,0) \mathrm{c}$ & 0,0 \\
\hline chlorimuron-ethyl & 15,0 & ALS & $1,8(7,5) \mathrm{d}$ & $10,5(18,7) \mathrm{hi}$ & $4,8(12,6)$ ef & $0,0(0,0) \mathrm{c}$ & 0,0 \\
\hline ethoxysulfuron & 150,0 & ALS & $2,8(9,4)$ bcd & $10,0(18,4)$ hij & $4,5(12,2)$ ef & $0,0(0,0) \mathrm{c}$ & 0,0 \\
\hline halosulfuron & 112,5 & ALS & $3,3(10,3)$ bcd & $4,5(11,3)$ ijk & $0,0(0,0) \mathrm{g}$ & $0,0(0,0) \mathrm{c}$ & 0,0 \\
\hline iodosulfuron-methyl & 10,0 & ALS & $3,0(9,9)$ bcd & $25,0(30,0)$ def & $10,0(18,4) \mathrm{de}$ & $0,0(0,0) \mathrm{c}$ & 0,0 \\
\hline metsulfuron-methyl & 2,4 & ALS & $4,8(11,8)$ bcd & $17,3(22,9) \mathrm{gh}$ & $6,8(14,5)$ ef & $0,0(0,0) \mathrm{c}$ & 0,0 \\
\hline nicosulfuron & 40,0 & ALS & $2,5(8,9) \mathrm{cd}$ & $11,3(19,2) \mathrm{hi}$ & $16,3(23,6) \mathrm{cd}$ & $0,0(0,0) \mathrm{c}$ & 0,0 \\
\hline pyrithiobac-sodium & 140,0 & ALS & $3,3(10,1)$ bcd & $40,0(39,0)$ bc & $36,3(36,9) b$ & $6,3(14,3) \mathrm{b}$ & 0,0 \\
\hline trifloxysulfuron-sodium & 22,5 & ALS & $3,0(9,9)$ bcd & $27,5(31,6) \mathrm{cde}$ & $7,5(15,7)$ ef & $0,0(0,0) \mathrm{c}$ & 0,0 \\
\hline $2,4-\mathrm{D}$ & 720,0 & Auxina & $1,8(7,5) \mathrm{d}$ & $0,8(4,3) \mathrm{kl}$ & $0,0(0,0) \mathrm{g}$ & $0,0(0,0) \mathrm{c}$ & 0,0 \\
\hline quinclorac & 375,0 & Auxina & $3,3(10,2)$ bcd & $4,0(10,7) \mathrm{jk}$ & $0,0(0,0) \mathrm{g}$ & $0,0(0,0) \mathrm{c}$ & 0,0 \\
\hline atrazina & $1.250,0$ & Fotossistema II & $4,0(11,4)$ bcd & $15,0(21,9) \mathrm{gh}$ & $3,0(9,8) \mathrm{f}$ & $0,0(0,0) \mathrm{c}$ & 0,0 \\
\hline bentazon & 600,0 & Fotossistema II & $2,8(9,4)$ bcd & $1,8(7,4) \mathrm{kl}$ & $0,0(0,0) \mathrm{g}$ & $0,0(0,0) \mathrm{c}$ & 0,0 \\
\hline linuron & $1.350,0$ & Fotossistema II & $6,3(14,3) b$ & $53,8(47,2)$ a & $63,8(53,0) a$ & $0,0(0,0) \mathrm{c}$ & 0,0 \\
\hline fomesafen & 187,5 & Protox & $2,8(9,4)$ bcd & $2,5(8,7) \mathrm{k}$ & $0,0(0,0) \mathrm{g}$ & $0,0(0,0) \mathrm{c}$ & 0,0 \\
\hline lactofen & 120,0 & Protox & $4,5(11,7)$ bcd & $16,8(22,0) \mathrm{h}$ & $5,3(12,7)$ ef & $0,0(0,0) \mathrm{c}$ & 0,0 \\
\hline oxadiazon & 600,0 & Protox & $6,0(13,7) b c$ & $11,8(18,2)$ hij & $3,5(10,5) \mathrm{f}$ & $0,0(0,0) \mathrm{c}$ & 0,0 \\
\hline oxyfluorfen & 720,0 & Protox & $22,5(28,1) \mathrm{a}$ & $47,5(43,6) a b$ & $20,0(26,4) \mathrm{c}$ & $0,0(0,0) \mathrm{c}$ & 0,0 \\
\hline$\overline{\mathrm{F}}_{\text {Tratamento }}$ & & & $7,626^{* *}$ & $22,675^{* *}$ & $50,679^{* *}$ & $3,439 * *$ & - \\
\hline $\mathrm{F}_{\text {Bloco }}$ & & & $3,082^{*}$ & $4,036^{* *}$ & $2,337^{\mathrm{ns}}$ & $0,910^{\mathrm{ns}}$ & -- \\
\hline CV $(\%)$ & & & 33,26 & 25,06 & 26,82 & 366,67 & - \\
\hline d.m.s. & & & 5,071 & 8,043 & 6,312 & 11,354 & -- \\
\hline
\end{tabular}

1/ Adicionou-se o adjuvante Assist $\left(0,5 \% \mathrm{v} \mathrm{v}^{-1}\right)$ à calda de aplicação. ${ }^{2 /}$ dados originais foram transformados em arco seno $\sqrt{\mathrm{x}} / 100$ (entre parênteses). Médias seguidas de mesma letra, nas linhas, não diferem estatisticamente entre si a 5\% de probabilidade pelo teste LSD.

** significativo a $1 \%$ de probabilidade; * significativo a $5 \%$ de probabilidade; ${ }^{\text {ns }}$ não significativo.

apresentou sintomas de fitointoxicação promovidos pelos herbicidas avaliados.

Conforme verificado neste trabalho, Christoffoleti \& Aranda (2001), avaliando a seletividade dos herbicidas halosulfuron $\left(112,5 \mathrm{~g} \mathrm{ha}^{-1}\right)$ e 2,4-D (2.010 $\left.\mathrm{g} \mathrm{ha}^{-1}\right)$ na grama $Z$. japonica, também observaram sintomas leves de fitointoxicação por um período de até 50 DAA; entretanto, verificaram que o oxadiazon $\left(1.000 \mathrm{~g} \mathrm{ha}^{-1}\right)$ promoveu sintoma moderado para o mesmo período de avaliação, sendo considerados níveis de fitointoxicação aceitáveis na prática. Esses pesquisadores ressaltaram que uma das prováveis razões da seletividade dos herbicidas pós-emergentes às gramas do gênero Zoyzia é decorrente do formato das folhas, que são normalmente estreitas e curtas, quando comparadas com as folhas das demais variedades de gramas dessa forma, essa característica dificulta a retenção do herbicida na superfície foliar.

Verificou-se que a densidade de inflorescências não foi influenciada pelos herbicidas chlorimuron-ethyl, bispyribac-sodium, ethoxysulfuron e fluazifop-p-butil. Contudo, apenas os inibidores da ALS iodosulfuronmethyl, pyrithiobac-sodium e trifloxysulfuronsodium estimularam a emissão de inflorescências em cerca de 100,5, 100,7 e 108,0\%, respectivamente, em relação à testemunha aos 49 DAA (Tabela 5). Os demais herbicidas reduziram a densidade de inflorescência em até $89,7 \%$, aos 49 DAA.

Com relação à altura das inflorescências, apenas o lactofen, o fluazifop-p-butil e o metsulfuron-methyl promoveram reduções na ordem de $32,1,34,0$ e 34,0\%, respectivamente, em relação à testemunha.

Para a altura da grama, observou-se que os herbicidas fluazifop-p-butil e sethoxydim (Accase), metsulfuron-methyl e nicosulfuron (ALS), 2,4-D e quinclorac (auxina), o bentazon (fotossistema II), fomesafen e lactofen (Protox) influenciaram o crescimento do gramado, promovendo reduções de até $43,8 \%$, comparandose com a testemunha aos 49 DAA. 
Tabela 5 - Valores médios de número de inflorescência, altura da planta, altura de inflorescência e massa seca do gramado de Z. japonica, após a aplicação de diferentes herbicidas, aos 49 DAA. Botucatu-SP, 2006

\begin{tabular}{|c|c|c|c|c|c|c|}
\hline \multirow[t]{2}{*}{ Tratamento } & Dose & \multirow{2}{*}{$\begin{array}{l}\text { Mecanismo } \\
\text { de ação }\end{array}$} & Inflorescência & $\begin{array}{c}\text { Altura da } \\
\text { inflorescência }\end{array}$ & $\begin{array}{c}\text { Altura da } \\
\text { grama }\end{array}$ & \multirow{2}{*}{$\begin{array}{c}\text { Massa seca } \\
\left(\mathrm{kg} \mathrm{ha}^{-1}\right)\end{array}$} \\
\hline & $\left(\right.$ g i.a. ha $\left.^{-1}\right)$ & & $\left(\text { número } \mathrm{m}^{-2}\right)^{2 /}$ & \multicolumn{2}{|c|}{$(\mathrm{cm})$} & \\
\hline Testemunha & --- & --- & $187,0(13,5)$ bc & 6,6 abcd & $7,3 \mathrm{abc}$ & 749,0 bcdef \\
\hline fluazifop-p-butil & 125,0 & Accase & $137,5(11,5) \mathrm{cd}$ & 4,4 e & $5,0 \mathrm{~d}$ & $334,6 \mathrm{~g}$ \\
\hline sethoxydim ${ }^{1 /}$ & 276,0 & Accase & $19,3(4,0) \mathrm{g}$ & $5,3 \mathrm{cde}$ & $4,8 \mathrm{~d}$ & $981,8 \mathrm{~b}$ \\
\hline bispyribac-sodium & 25,0 & ALS & $107,8(10,3)$ cde & 5,8 bcde & 5,8 bcd & $1409,3 \mathrm{a}$ \\
\hline chlorimuron-ethyl & 15,0 & ALS & $261,3(15,5)$ ab & 6,5 abcd & $8,5 \mathrm{a}$ & 549,3 efg \\
\hline ethoxysulfuron & 150,0 & ALS & $254,3(15,7)$ ab & 6,8 abcd & $7,4 \mathrm{ab}$ & 571,6 defg \\
\hline halosulfuron & 112,5 & ALS & $67,8(7,9)$ defg & 5,3 cde & $5,5 \mathrm{~cd}$ & 819,9 bcde \\
\hline iodosulfuron-methyl & 10,0 & ALS & $375,0(19,3)$ a & $8,0 \mathrm{a}$ & $8,6 \mathrm{a}$ & $1015,7 \mathrm{~b}$ \\
\hline metsulfuron-methyl & 2,4 & ALS & $77,5(8,4)$ def & $4,4 \mathrm{e}$ & $4,6 \mathrm{~d}$ & $1052,9 \mathrm{ab}$ \\
\hline nicosulfuron & 40,0 & ALS & $72,8(7,9)$ defg & $4,9 \mathrm{de}$ & $4,8 \mathrm{~d}$ & 535,9 efg \\
\hline pyrithiobac-sodium & 140,0 & ALS & $375,3(19,4)$ a & $6,9 a b c$ & $7,3 a b c$ & 492,8 efg \\
\hline trifloxysulfuron-sodium & 22,5 & ALS & $389,0(19,3)$ a & $7,5 \mathrm{ab}$ & $7,6 \mathrm{a}$ & 571,8 defg \\
\hline 2,4-D & 720,0 & Auxina & $38,0(6,1) \mathrm{fg}$ & 6,1 abcde & $5,3 \mathrm{~d}$ & 450,4 efg \\
\hline quinclorac & 375,0 & Auxina & $37,8(6,0) \mathrm{fg}$ & 4,9 de & $4,1 \mathrm{~d}$ & 452,4 efg \\
\hline atrazina & $1.250,0$ & Fotossistema II & $49,3(6,8)$ efg & 5,9 bcde & $5,5 \mathrm{~cd}$ & $955,6 \mathrm{bc}$ \\
\hline bentazon & 600,0 & Fotossistema II & $42,0(6,4)$ efg & 5,3 cde & $5,3 \mathrm{~d}$ & $436,4 \mathrm{fg}$ \\
\hline linuron & $1.350,0$ & Fotossistema II & $100,0(9,1)$ def & $5,5 \mathrm{cde}$ & $7,3 a b c$ & 1396,8 a \\
\hline fomesafen & 187,5 & Protox & $76,3(8,5)$ def & 5,1 cde & $5,4 \mathrm{~d}$ & 703,9 bcdefg \\
\hline lactofen & 120,0 & Protox & $31,8(4,2) \mathrm{g}$ & $4,5 \mathrm{e}$ & $5,3 \mathrm{~d}$ & $969,7 \mathrm{bc}$ \\
\hline oxadiazon & 600,0 & Protox & $82,5(8,9)$ def & 5,9 bcde & $5,5 \mathrm{~cd}$ & 610,5 cdefg \\
\hline oxyfluorfen & 720,0 & Protox & $48,0(6,4)$ efg & 5,1 cde & 5,8 bcd & 923,6 bcd \\
\hline $\mathrm{F}_{\text {Tratamento }}$ & & & $12,283^{* *}$ & $2,088^{* *}$ & $4,263^{* *}$ & $5,501 * *$ \\
\hline $\mathrm{F}_{\text {Bloco }}$ & & & $4,178^{* *}$ & $1,128^{\mathrm{ns}}$ & $4,295^{* *}$ & $2,672 *$ \\
\hline CV $(\%)$ & & & 27,51 & 24,30 & 21,27 & 34,34 \\
\hline d.m.s. & & & 3,989 & 1,972 & 1,808 & 369,655 \\
\hline
\end{tabular}

${ }^{1 /}$ Adicionou-se o adjuvante Assist $\left(0,5 \% \mathrm{v} \mathrm{v}^{-1}\right)$ à calda de aplicação. ${ }^{2 /}$ dados originais foram transformados em $\sqrt{\mathrm{x}}+0,5$ (entre parênteses). Médias seguidas de mesma letra, nas linhas, não diferem estatisticamente entre si a $5 \%$ de probabilidade pelo teste LSD.

** significativo a $1 \%$ de probabilidade; $*$ significativo a $5 \%$ de probabilidade; ${ }^{\text {ns }}$ não significativo.

É importante destacar que os herbicidas 2,4-D, quinclorac, bentazon e fomesafen, além de proporcionarem reduções na altura da grama, também apresentaram sintomas de fitointoxicação considerados leves $(\leq 10,0 \%)$ por até 7 DAA. No entanto, as reduções na altura da grama promovidas por esses herbicidas não se refletiram na produção de massa seca das plantas, uma vez que apresentaram valores estatisticamente semelhantes aos da testemunha (Tabelas 4 e 5). Assim, observouse ainda que, entre os herbicidas avaliados, apenas o bispyribac-sodium e o linuron apresentaram produção de massa seca em torno de 88,2 e $86,5 \%$ superior em relação à testemunha. Entretanto, estes herbicidas prejudicaram o aspecto visual do gramado, devido aos elevados sintomas de fitointoxicação, por até 14 DAA.

Considerando os herbicidas que proporcionaram fitointoxicação leve $(\leq 10,0 \%)$ por no máximo uma semana e que não influenciaram a produção da massa seca das plantas, destacaram-se o halosulfuron, o 2,4-D, o quinclorac, o bentazon e o fomesafen. Da mesma forma, para os dados da produção da massa seca, os herbicidas fluazifop-p-butil, sethoxydim, iodosulfuron-methyl, metsulfuron-methyl, nicosulfuron, pyrithiobac-sodium, trifloxysulfuron-sodium, lactofen e oxyfluorfen não diferiram da testemunha, porém promoveram fitointoxicação moderada a severa por um período de 14 a 26 DAA.

Assim, de acordo com Christoffoleti \& Aranda (2001), que afirmaram que a seletividade de herbicidas aos diversos tipos de grama está em função principalmente da espécie de grama e do tipo herbicida aplicado, bem como da dose utilizada, pode-se concluir que, dos herbicidas avaliados no presente estudo, os que apresentaram potencial de seletividade para o gramado de $S$. secundatum 
foram: os inibidores da ALS chlorimuron-ethyl, ethoxysulfuron, halosulfuron, iodosulfuronmethyl e metsulfuron-methyl; o mimetizador de auxina 2,4-D; os inibidores do fotossistema II atrazina e bentazon; e os inibidores da Protox fomesafen, lactofen e oxadiazon. Para o gramado de $Z$. japonica, os herbicidas que apresentaram potencial de seletividade foram: os inibidores da ALS chlorimuron-ethyl, ethoxysulfuron, halosulfuron, metsulfuronmethyl e nicosulfuron; os mimetizadores de auxina 2,4-D e quinclorac; os inibidores do fotossistema II atrazina e bentazon; e os inibidores da Protox fomesafen, lactofen e oxadiazon.

Portanto, esses herbicidas podem ser utilizados tanto em programas de controle de plantas daninhas em gramados, fornecendo opções de mecanismos de ação distintos para a prevenção de surgimento de plantas resistentes a herbicidas, quanto como reguladores vegetais. Contudo, mais estudos são necessários com relação ao uso desses herbicidas como reguladores vegetais, sobretudo no que se refere a doses e momento de aplicação. Da mesma forma, Busey (2003) afirma que mais estudos devem ser realizados no que diz respeito às consequências a longo prazo da aplicação de herbicidas na mesma área sobre o banco de sementes e seus efeitos com a interação com métodos culturais de controle de plantas daninhas utilizados em gramados.

\section{LITERATURA CITADA}

BRECKE, B. J.; UNRUH, B.; DUSKY, J. A. Torpedograss (Panicum repens) control with quinclorac in bermudagrass (Cynodon dactylon x C. transvaalensis) turf. Weed Technol., v. 15, n. 4 , p. $732-736,2001$

BUSEY, P. Cultural management of weed in turfgrass: a review. Crop Sci., v. 43, n. 6, p. 1899-1911, 2003.

BUSEY, P.; JOHNSTON, D. L. Impact of cultural factors on weed populations in St. Augustinegrass turf. Weed Sci., v. 54, n. 5, p. 961-967, 2006.

COSTA, N. V. et al. Características morfológicas de gramas em resposta à aplicação de trinexapac-ethyl.

Planta Daninha, v. 27, n. 1, p. 113-122, 2009.

CHRISTOFFOLETI, P. J.; ARANDA, A. N. Seletividade de herbicidas a cinco tipos de gramas. Planta Daninha, v. 19, n. 2, p. 273-278, 2001.
DVORANEN, E. C. et al. Nodulação e crescimento de variedades de soja RR sob aplicação de glyphosate, fluazifopp-butyl e fomesafen. Planta Daninha, v. 26, n. 3, p. 619-625, 2008.

FAGERNESS, M. J.; YELVERTON, F. H.; COOPER, R. J. Bermudagrass [Cynodon dactylon (L.) Pers.] and Zoysiagrass (Zoysia japonica) establishment after preemergente herbicide applications. Weed Technol., v. 16, n. 3, p. 597-602, 2002.

FREITAS, F. C. L. et al. Eficiência do triclopyr no controle de plantas daninhas em gramado (Paspalum notatum)

Planta Daninha, v. 21, n. 1, p. 159-164, 2003.

GAZZIERO, D. L. P. et al. Variabilidade no grau de resistência de capim-marmelada (Brachiaria plantaginea) aos herbicidas clethodim, tepraloxydim e sethoxydim. Planta Daninha, v. 22, n. 3 , p. $397-402,2004$

JOHNSON, B. J.; CARROW, R. N. Tolerance of zoysiagrass (Zoysia spp.) cultivars to preemergence herbicides. Weed Technol., v. 13, n. 4, p. 706-712, 1999.

MACIEL, C. D. G. et al. Comportamento vegetativo e reprodutivo da grama-batatais (Paspalum notatum Flügge) submetida à aplicação de subdoses dos herbicidas clethodim e sethoxydim. In: SIGRA - SIMPÓSIO SOBRE GRAMADOS - "ATUALIDADES \& PERSPECTIVAS", 3., 2006, Botucatu. Anais... Botucatu: FCA/UNESP, 2006. 4 p. CD-ROM

McCARTY, L. B.; PORTER, W.; COLVIN, D. L. Sod regrowth of St. Augustinegrass after preemergence herbicide application. Agron. J., v. 87, n. 3, p. 503-507, 1995.

PIMENTA, C. H. Produção de gramas. In: SIGRASIMPÓSIO SOBRE GRAMADOS - "PRODUÇÃO, IMPLANTAÇÃO E MANUTENÇÃO”, 1., 2003, Botucatu Anais... Botucatu: FCA/UNESP, 2003. 7 p. CD-ROM

RODRIGUES, B. N.; ALMEIDA, F. S. Guia de herbicidas. 5.ed. Londrina: 2005. 592 p.

SANTOS, J. B. et al. Ação de herbicidas sobre o crescimento de estirpes de Rhizobium tropici. Planta Daninha, v. 24, n. 3, p. $457-465,2006$.

SHIM, S. R.; JOHNSON, B. J. Response of creeping bentgrass to spring-applied herbicides. Hortscience, v. 27, n. 3, p. 237$239,1992$.

SOCIEDADE BRASILEIRA DA CIÊNCIA DAS PLANTAS DANINHAS - SBCPD. Procedimentos para instalação, avaliação e análise de experimentos com herbicidas Londrina: 1995. 42 p.

TURNER, D. L.; SHARPE, S. S.; DICKENS, R. Herbicide effects on tensile strength and rooting of centipedegrass sod. Hortscience, v. 25, n. 5, p. 541-544, 1990.

WILLARD, T. R.; PEACOCK, C. M.; SHILLING, D. G. Photosynthesis as an index of turfgrass growth following application of herbicides. Hortscience, v. 25, n. 4, p. 451-453, 1990. 